\title{
Benchmark Data for Evaluation of Aeroacoustic Propagation Codes with Grazing Flow
}

\author{
M. G. Jones, ${ }^{*}$ W. R. Watson ${ }^{\dagger}$ and T. L. Parrott ${ }^{\ddagger}$ \\ NASA Langley Research Center, Hampton, VA 23681-2199, USA
}

\begin{abstract}
INCREASED understanding of the effects of acoustic treatment on the propagation of sound through commercial aircraft engine nacelles is a requirement for more efficient liner design. To this end, one of NASA's goals is to further the development of duct propagation and impedance eduction codes. A number of these codes have been developed over the last three decades. These codes are typically divided into two categories: (1) codes that use the measured complex acoustic pressure field to educe the acoustic impedance of treatment that is positioned along the wall of the duct, and (2) codes that use the acoustic impedance of the treatment as input and compute the sound field throughout the duct. Clearly, the value of these codes is dependent upon the quality of the data used for their validation. Over the past two decades, data acquired in the NASA Langley Research Center Grazing Incidence Tube have been used by a number of researchers for comparison with their propagation codes. Many of these comparisons have been based upon Grazing Incidence Tube tests that were conducted to study specific liner technology components, and were incomplete for general propagation code validation. Thus, the objective of the current investigation is to provide a quality data set that can be used as a benchmark for evaluation of duct propagation and impedance eduction codes. In order to achieve this objective, two parallel efforts have been undertaken. The first of these is the development of an enhanced impedance eduction code that uses data acquired in the Grazing Incidence Tube. This enhancement is intended to place the benchmark data on as firm a foundation as possible. The second key effort is the acquisition of a comprehensive set of data selected to allow propagation code evaluations over a range of test conditions.
\end{abstract}

\section{Nomenclature}

$\begin{array}{ll}c & \text { ambient sound speed, } \mathrm{m} / \mathrm{s} \\ H, L, W & \text { test section height, length and width, respectively, } \mathrm{m} \\ i & \sqrt{-1} \\ k & \text { free space wavenumber, } \mathrm{m}^{-1} \\ {[K(\zeta)]} & \text { sparse, complex, asymmetric matrix with order } 4 M N \\ L_{1}, L_{2} & \text { axial locations of leading and trailing edges of liner, respectively, } \mathrm{m} \\ m & \text { mode order in spanwise direction } \\ M(x, y, z) & \text { local Mach number } \\ M_{0} & \text { uniform flow (average) Mach number } \\ N, M & \text { node numbers in axial and vertical directions, respectively } \\ p & \text { complex acoustic pressure, Pa } \\ p_{s} & \text { acoustic pressure at source plane, Pa } \\ P_{m} & \text { measured acoustic pressure, Pa } \\ x, y, z & \text { axial, vertical and spanwise locations, respectively, } \mathrm{m} \\ x_{i} & \text { wall measurement axial location, } \mathrm{m}\end{array}$

${ }^{*}$ Research Scientist, Research \& Technology Directorate, Aeroacoustics \& Structural Acoustics Branch, Mail Stop 463; Michael.G.Jones@NASA.GOV. Senior member of AIAA.

${ }^{\dagger}$ Senior Research Scientist, Research \& Technology Directorate, Computational Aerosciences Branch, Mail Stop 128; Willie.R.Watson@NASA.GOV. Senior member of AIAA.

${ }^{\ddagger}$ Senior Research Scientist, Research \& Technology Directorate, Aeroacoustics \& Structural Acoustics Branch, Mail Stop 463; Tony.L.Parrott@NASA.GOV. 
Symbols:

$\begin{array}{ll}\rho & \text { ambient density, } \mathrm{kg} / \mathrm{m}^{3} \\ \theta & \text { normalized acoustic resistance } \\ \chi & \text { normalized acoustic reactance } \\ \zeta & \theta+i \chi, \text { normal incidence acoustic impedance, normalized by } \rho c \\ \omega & \text { angular frequency, } \mathrm{s}^{-1} \\ \zeta_{\mathrm{e}} & \text { exit plane impedance }\end{array}$

Abbreviations:

2D-FEM

GIT

Q3D-FEM

Subscripts:

FEM

Meas

\section{2-D Finite Element Method \\ Grazing Incidence Tube \\ Quasi-3D Finite Element Method}

finite element method result

measured results

\section{Introduction}

NCREASED understanding of acoustic liner effects on sound propagation through aircraft engine nacelles is required Ifor more efficient liner design. Numerous aeroacoustic duct propagation codes have been developed over the last three decades, ${ }^{1-9}$ by the current authors as well as many others. These codes use measured acoustic pressures to educe the liner impedance or, conversely, use the liner impedance to compute the sound field throughout the duct. The value of these codes is critically dependent upon rigorous validation. Over the last two decades, a number of code validations have been based upon NASA Grazing Incidence Tube (GIT) data. ${ }^{2-8}$ However, the data used for many of these validations were not intended for this purpose, and were therefore incomplete. The objective of the current investigation is to provide a complete data set that can conveniently be used as a benchmark for evaluating duct propagation and impedance eduction codes. Two efforts have been undertaken to achieve this objective. The first is the validation of an enhanced impedance eduction code. The second is to provide a comprehensive set of Grazing Incidence Tube data acquired over a range of test conditions, together with the resultant impedances (educed with this enhanced code), in a manner suitable for validations of other propagation codes.

For the past six years, a number of studies have demonstrated the use of a 2-D finite element method (2D-FEM) ${ }^{2-5}$ for impedance eduction with complex acoustic pressure data acquired in the GIT. This method iterates on the numerical solution to either the 2-D convected wave equation ${ }^{2,4,5}$ or the linearized equations of mass and momentum ${ }^{3}$ to determine the impedance that reproduces the measured complex acoustic pressure field at selected measurement locations throughout the duct. It uses the duct geometry, mean flow, measured exit impedance (for an assumed plane wave) and a plane wave source as data inputs. However, there is a need to extend the methodology to remove the zero-order mode limitation, and thus to extend the frequency range that can be evaluated in the GIT (the first higher-order mode cuts on at approximately $3.3 \mathrm{kHz}$ for the case of no flow in the GIT). The eventual goal is to implement a code that extends the frequency range up to $10.0 \mathrm{kHz}$ (by including higher-order modes) and accommodates non-parallel shear flow profiles. This will require a fully 3-D methodology, and efforts are underway at NASA to develop such a code. In the interim, a quasi-3D finite element methodology (Q3D-FEM) is presented that greatly reduces the computational time and memory requirements by reducing the 3-D problem to a series of 2-D calculations. The Q3D-FEM is valid in constant area ducts for which the acoustic field is separable in one cross-sectional direction.

A ceramic tubular liner is used for this study that is nearly linear with respect to mean flow velocity and sound pressure level (SPL), and provides an impedance spectrum that varies over a range typically observed in aircraft engine nacelle liners. Acoustic pressure data measured in the GIT are used to educe the liner impedance with both methodologies (2D-FEM and Q3D-FEM). Previous studies have shown the 2D-FEM to provide quality results. Thus, excellent agreement between results achieved using the two methodologies demonstrates the suitability of the quasi-3D methodology for test conditions where the 2-D method is appropriate (a plane wave source is used), and increases confidence that the quasi-3D approach will be suitable in the presence of higher-order modes. Each methodology is then used to provide educed impedances for a range of mean flow velocities (Mach 0.0 to Mach 0.5 ), source frequencies ( 0.5 to 3.0 $\mathrm{kHz}$ ), and source sound pressure levels (SPLs of 120 to $140 \mathrm{~dB}$ ). For completeness, the measured acoustic pressures (SPL and phase) on the wall of the duct opposite the liner, the exit plane impedance spectrum, and representative mean flow profiles in the GIT are also provided. 


\section{Analysis}

TIGURE 1 is a schematic of the three-dimensional flow duct and Cartesian coordinate system used in this study. The Fource and exit planes of the duct are located at $x=0$ and $x=L$, respectively, and the two side-walls and lower wall of the duct are rigid. The test liner constitutes the portion of the upper wall between $L_{1} \leq x \leq L_{2}$, and the wall is rigid outside of this region. The sound absorbing material is assumed to present a uniform and local-reacting normalized impedance, $\zeta$, to the aeroacoustic field in the duct. It is further assumed that the acoustic pressure field on the wall opposite the test liner is known from acoustic measurements at a set of discrete locations. Finally, there is a assumed to be a uniform mean flow from left to right with subsonic Mach number, $M_{0}$. Although this figure depicts a model of the aft-fan duct (i.e., sound and mean flow are in the same direction), inlet duct modeling (i.e., flow and sound in opposite directions) is achieved by reversing the sign on the Mach number. The problem at hand is to determine the unknown impedance, $\zeta$, of the test liner in the presence of the uniform mean flow.

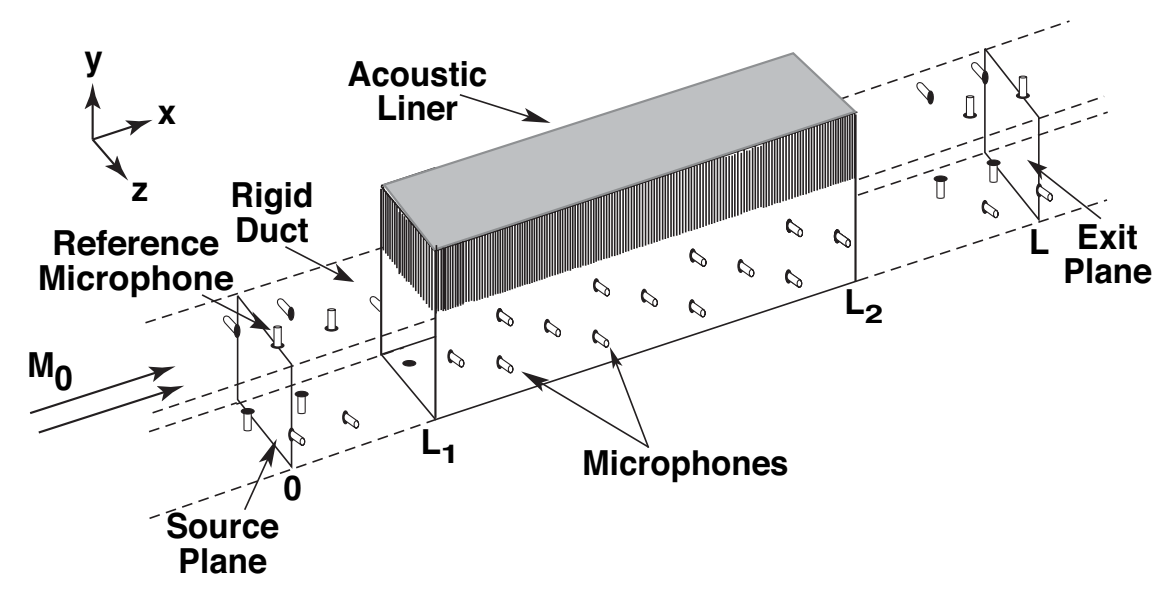

Figure 1. Sketch of Grazing Incidence Tube (GIT) Test Window.

\section{A. Differential Equation and Boundary Conditions}

$\mathrm{A}^{\text {Coustic pressure disturbances in the duct are governed by the convected Helmholtz equation }}$

$$
\left(1-M_{0}^{2}\right) \frac{\partial^{2} p}{\partial x^{2}}+\frac{\partial^{2} p}{\partial y^{2}}+\frac{\partial^{2} p}{\partial z^{2}}-2 i k M_{0} \frac{\partial p}{\partial x}+k^{2} p=0
$$

where a time dependence of the form $e^{i \omega t}$ has been assumed. To obtain a unique solution to the above equation, boundary conditions must be prescribed. These include a wall boundary condition at the duct walls, a source boundary condition at the source plane, and an exit boundary condition at the exit plane of the duct.

The local-reacting wall impedance boundary condition for the liner, as presented by Myers, ${ }^{10}$ gives

$$
\frac{\partial p}{\partial y}=i k\left(\frac{p}{\zeta}\right)+2 M_{0} \frac{\partial}{\partial x}\left(\frac{p}{\zeta}\right)+\frac{M_{0}^{2}}{i k} \frac{\partial^{2}}{\partial x^{2}}\left(\frac{p}{\zeta}\right)
$$

The above wall impedance boundary condition is also valid along the rigid portion of the duct provided that the local-reacting impedance is set to infinity $(\zeta=\infty)$. At the source plane, the acoustic pressure is assumed known

$$
p=p_{s}
$$

while at the duct termination, a variety of boundary conditions that render the boundary value problem on the acoustic pressure field well-posed can be implemented.

When only the plane wave mode is cut on at the exit plane, it is convenient to specify an exit impedance condition such that the boundary condition will be of the form

$$
\frac{\partial p}{\partial x}=-\frac{i k p}{\left(M_{0}+\zeta_{\mathrm{e}}\right)}
$$


The exit impedance boundary condition (equation 4 ) with constant coefficients is easily implemented into the impedance eduction procedure. Further, the exit impedance (i.e., $\zeta_{\mathrm{e}}$ ) is easily measured using the two-microphone method ${ }^{11}$ based on data acquired in the GIT side-wall with a rotating plug.

\section{B. Quasi 3-D Theory}

A $\mathrm{S}$ mentioned earlier, the eventual goal is to implement a 3-D propagation code that accounts for higher-order A modes (and thus extends the frequency range up to $10.0 \mathrm{kHz}$ ) and non-parallel shear flow profiles in the GIT. In the interim, a quasi-3D impedance eduction model is provided that adequately represents many of the essential features that will occur in a fully 3 -D computation. This model greatly reduces the computational time and memory required to obtain the numerical solution by reducing the 3-D problem to a series of 2-D calculations. The quasi-3D model is applicable when the solution in the spanwise direction is separable (e.g., when one or both side-walls of the duct are lined with uniform acoustic treatment and the mean flow is independent of the spanwise direction). When the side-walls of the duct are rigid, as in the duct depicted in fig. 1, the acoustic pressure field and sound source may be expanded into the following Fourier series of hard wall duct modes

$$
\begin{aligned}
p(x, y, z) & =\sum_{m=0}^{m=\infty} P_{m}(x, y) \cos \left(\frac{m \pi z}{W}\right) \\
p_{s}(y, z) & =\sum_{m=0}^{m=\infty} G_{m}(y) \cos \left(\frac{m \pi z}{W}\right)
\end{aligned}
$$

where the function $P_{m}$ satisfies the partial differential equation

$$
\left(1-M_{0}^{2}\right) \frac{\partial^{2} P_{m}}{\partial x^{2}}+\frac{\partial^{2} P_{m}}{\partial y^{2}}-2 i k M_{0} \frac{\partial P_{m}}{\partial x}+\left[k^{2}-\left(\frac{m \pi}{W}\right)^{2}\right] P_{m}=0
$$

the sound source boundary condition

$$
P_{m}(0, y)=G_{m}(y), m=0,1,2, \ldots \infty
$$

the wall impedance boundary condition

$$
\frac{\partial P_{m}}{\partial y}=i k\left(\frac{P_{m}}{\zeta}\right)+2 M_{0} \frac{\partial}{\partial x}\left(\frac{P_{m}}{\zeta}\right)+\frac{M_{0}^{2}}{i k} \frac{\partial^{2}}{\partial x^{2}}\left(\frac{P_{m}}{\zeta}\right)
$$

and the exit plane condition

$$
\frac{\partial P_{m}}{\partial x}=-i k \frac{P_{m}}{\left(M_{0}+\zeta_{\mathrm{e}}\right)}
$$

Equations (7)-(10) constitute a well-posed boundary-value problem that can be solved to uniquely determine the lower wall acoustic pressure, $P_{m}(x, 0)$, for a given test liner impedance. Conversely, if the lower wall acoustic pressure distribution is known, this boundary-value problem can be iteratively solved to determine the liner impedance that reproduces the known lower wall acoustic pressure distribution. This procedure is often referred to in the literature as impedance eduction, and the resultant impedance is an estimate of the test liner impedance. Closed form solutions for test liner impedances that reproduce a known lower wall acoustic pressure distribution are available only for infinitely long ducts with uniform wall linings. Thus, a numerical procedure is needed to educe the test liner impedance.

\section{Impedance Eduction Technique}

THE impedance eduction technique chosen to solve equations (7)-(10) closely parallels that used in an earlier paper

for the zero-order spanwise mode (i.e., $m=0$ ). It is assumed that there are $N$ and $M$ evenly spaced nodes in the axial $(x)$ and vertical $(y)$ directions of the duct, respectively. A finite element method, with cubic polynomial basis functions and rectangular elements, is applied to obtain the lower wall acoustic pressure solution for a given liner impedance. A weak formulation is employed to incorporate the wall and exit impedance boundary conditions. The finite element method results in a discrete system of equations of the form

$$
[K(\zeta)]\{\Phi\}=\{B\}
$$


An asymmetric, parallel, direct sparse solver is used to obtain $\{\Phi\}$. This solver employs a compressed-column storage scheme to reduce storage overhead. Only the nonzero coefficients within the bandwidth of $[K(\zeta)]$ are stored, along with two pointer arrays that store the column numbers and starting indices of these nonzero coefficients. To achieve efficient solutions, the sparse solver uses two accelerators: equation reordering to reduce fill during the factorization of $[K(\zeta)]$ and parallelization. (The equation solver runs on multiple processors simultaneously.) Stewart's adaptation of the Davidon-Fletcher-Powell optimization algorithm is used to iteratively determine the unknown impedance, $\zeta$, by minimizing the objective function

$$
F(\zeta)=\sum_{i=1}^{N}\left[\left.P_{m}\left(x_{i}, 0\right)\right|_{\mathrm{FEM}}-\left.P_{m}\left(x_{i}, 0\right)\right|_{\mathrm{Meas}}\right]\left[\left.P_{m}^{*}\left(x_{i}, 0\right)\right|_{\mathrm{FEM}}-\left.P_{m}^{*}\left(x_{i}, 0\right)\right|_{\mathrm{Meas}}\right]
$$

where "*" denotes the complex conjugate of a quantity.

\section{Experimental Procedure}

\section{A. GIT Test Section}

THE GIT test section (fig. 1) has internal dimensions of $0.812 \mathrm{~m} \times 0.051 \mathrm{~m} \times 0.51 \mathrm{~m}$, and contains an axially-centered

test liner with a length of $0.406-\mathrm{m}$. Thus, the liner length-to-duct height ratio is eight. The surface of the test liner forms the upper wall for the flow duct. Elsewhere, the test section side-walls are rigid, with a source upstream of the liner and a near-anechoic termination downstream of the liner. Data acquired with the 31 microphones mounted on the lower wall (opposite the liner, see fig. 2) are used as input to the 2D-FEM and Q3D-FEM impedance eduction codes.

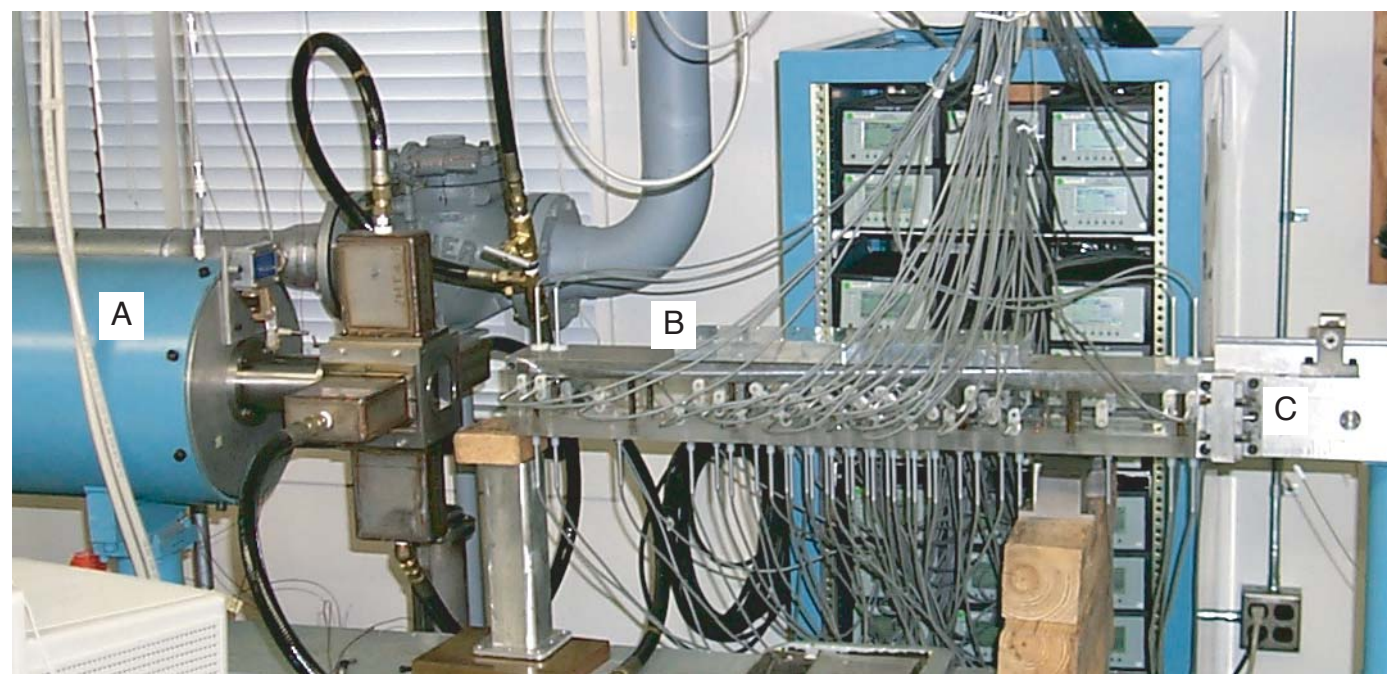
A. Air source plenum
B. Top-mounted liner fixture
C. Termination

Figure 2. Photograph of Grazing Incidence Tube.

Agilent analog-to-digital devices are used to simultaneously acquire the data for 48 channels, and a Cytec switch matrix is used to switch from one set of 48 microphones (reference microphone plus microphones 2 through 48 ) to another set of 48 microphones (reference microphone plus microphones 49 through 95). The reference microphone (one of the 95 flush-mounted microphones, located in the source plane) provides the common basis for both sets of measurements, such that measurements at all 95 locations can be properly compared. For the current investigation only the 31 microphones located on the wall opposite the liner are used in the impedance eduction process. For each data acquisition, 2000 averages on each microphone channel (blocks of 2048 data points per average) were used in the

current investigation. To reduce the influence of flow noise, a cross-spectrum signal extraction method ${ }^{12}$ was used to determine the amplitudes and phases at each of the microphone locations (relative to the amplitude and phase at the reference microphone location). 


\section{B. Ceramic Tubular Liner (CT57)}

ceramic tubular liner depicted in fig. 3 was used for this study. This liner has been shown to be nearly linear with A respect to mean flow velocity and SPL, and provides an impedance spectrum that varies over a range typically observed in aircraft engine nacelle liners. It consists of parallel, cylindrical channels embedded in a ceramic matrix. These channels, with diameters of $0.6 \mathrm{~mm}$, run perpendicular to the exposed surface and provide a surface porosity of $57 \%$. The $85.6 \mathrm{~mm}$-deep channels are rigidly terminated such that each is isolated from its neighbor to ensure a locally reacting structure. The channel diameter is small enough that the grazing flow effects are insignificant relative to internal viscous losses.

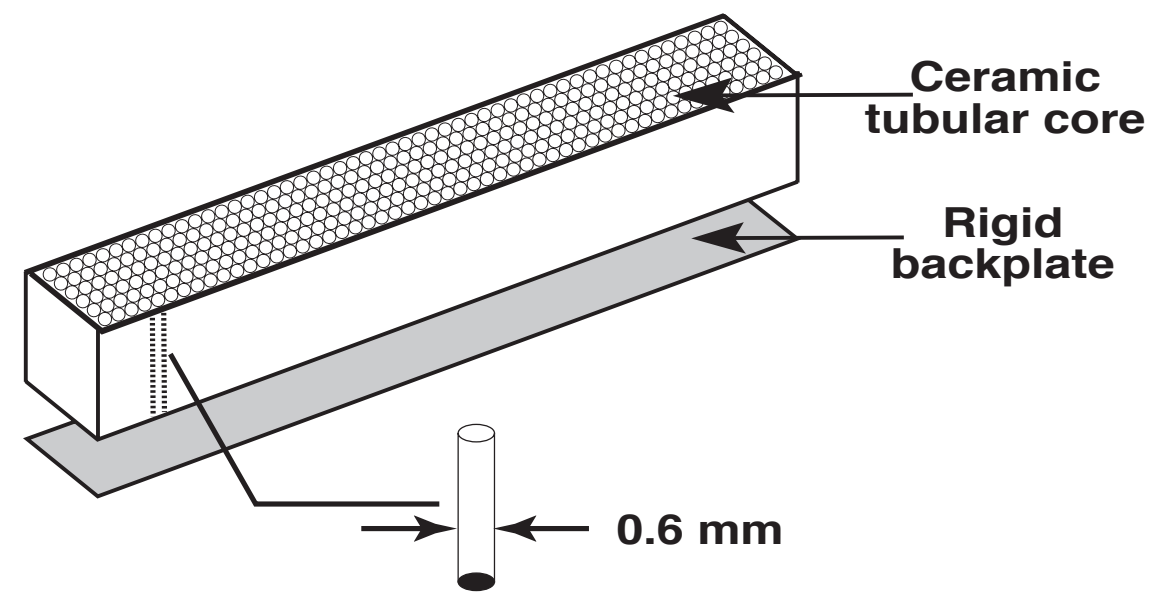

Figure 3. Sketch of ceramic tubular liner.

\section{Test Description}

$\mathrm{D}$ ETAILED mean flow profiles were acquired at three axial locations ( $x=203 \mathrm{~mm}, x=356 \mathrm{~mm}$ and $x=559 \mathrm{~mm}$ ) for this investigation. At each axial location, nine probes (eight pitot probes and one pitot-static probe) were vertically traversed across the test section to acquire a detailed survey ( 9 by 27 measurement array) across the cross-sectional plane (see fig. 4). Numerical integration was used to compute an average Mach number for the selected axial plane. Finally, the average Mach numbers for each of the three axial planes were averaged together to attain a "smeared" average Mach number for use in the 2D-FEM and Q3D-FEM impedance eduction codes. For the current investigation, this process was completed for set-point centerline Mach numbers of 0.1, 0.2, 0.3, 0.4 and 0.5 (determined using a pito-static tube located at approximately $x=860 \mathrm{~mm}$ ). However, the flow profiles measured for the set-point centerline Mach number of 0.5 were later discovered to cause leakage in the GIT, as evidenced by a loss of mass flow between the midstream $(x=356 \mathrm{~mm})$ and downstream $(x=559 \mathrm{~mm})$ measurement planes. Additional tests proved that the leakage is avoided if the set-point centerline Mach number is no greater than 0.475. Based on the profiles measured at set-point centerline Mach numbers up to 0.4, together with the upstream and midstream profiles for the set-point centerline Mach number of 0.5, the average Mach number for the set-point centerline Mach number of 0.475 was determined to be 0.4 .

The methodology for acquiring acoustic data with the GIT has been thoroughly described in a previous report. ${ }^{13}$ For the current investigation, acoustic tests were conducted for each the following settings:

1. Sound pressure levels (SPL) at the source plane ( $x=0.0 \mathrm{~mm}$, see fig. 1) of 120,130 and $140 \mathrm{~dB}$

2. Source frequencies (discrete tone source, one tone at a time) of 500 to $3000 \mathrm{~Hz}$, in steps of $100 \mathrm{~Hz}$

3. Set-point centerline Mach numbers of 0.0, 0.1, 0.2, 0.3, 0.4 and 0.475 (these correspond to average Mach numbers of $0.0,0.079,0.172,0.255,0.335$ and 0.400 , respectively)

A function generator was used to supply a sinusoidal signal at the desired test frequency to four 120-watt acoustic drivers (see fig. 5). The acoustic waves emitted from the acoustic drivers propagate from left to right across the surface of the test panel, and into a termination section designed to minimize reflections over the frequency range of interest. At each test frequency, the desired sound pressure level was set at the source plane (as measured by the reference 


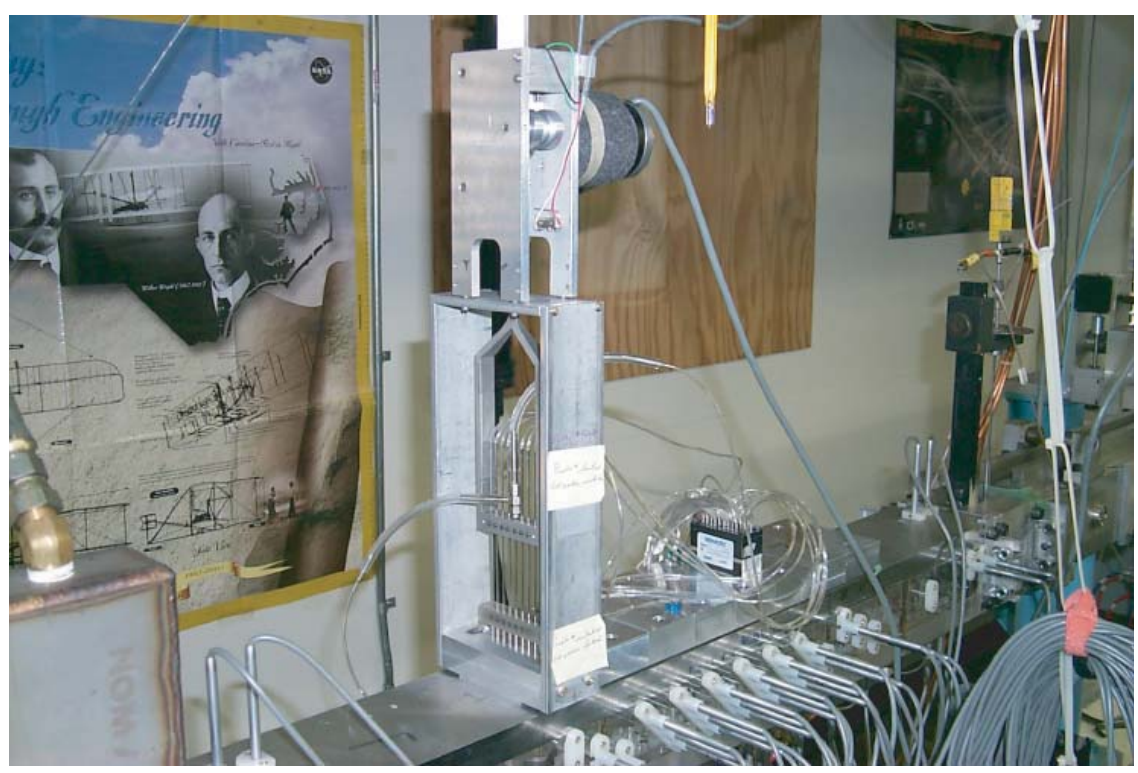

Figure 4. Photograph of 9-Probe Flow Measurement System.

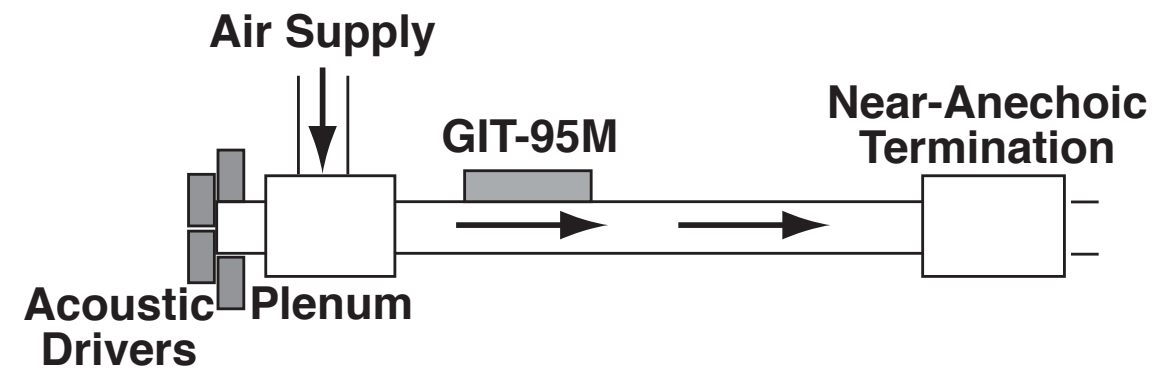

Figure 5. Sketch of Grazing Incidence Tube.

microphone). The data acquisition system described earlier was then used to record the microphone signals, and the data were stored for post-processing.

For each test condition, the data to be provided will include:

1. SPL at the source plane $(x=0.0 \mathrm{~mm})$

2. Impedance at the exit plane $(x=812.8 \mathrm{~mm})$

3. Complex acoustic pressure profile - SPL and phase (relative to that at $x=0.0 \mathrm{~mm}$ ) at each measurement location (31 microphones are located along the lower wall of the GIT, opposite of the acoustic liner)

4. Mean flow profiles measured along the length of the lined portion of the duct (at $x=203 \mathrm{~mm}, x=356 \mathrm{~mm}$ and $x=559 \mathrm{~mm})$

5. Liner impedances educed from the measured data using the 2D-FEM and Q3D-FEM codes

\section{Results and Discussion}

database consisting of six Mach numbers, twenty-six test frequencies, and three source sound pressure levels was complete database is available in electronic format upon request. 


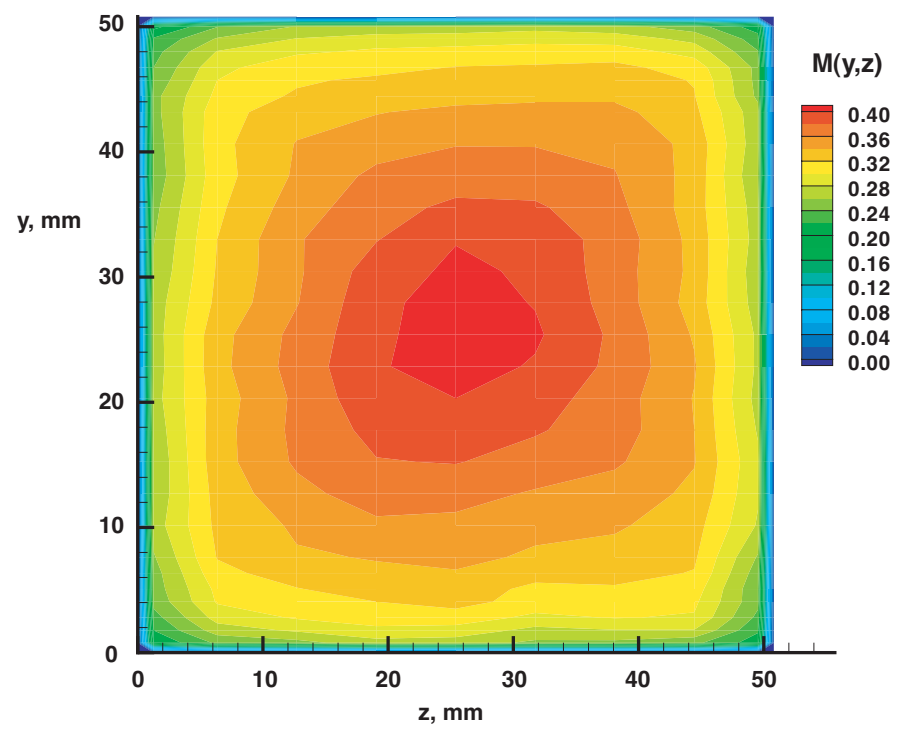

Figure 6. One of three $M(y, z)$ contour plots (at $x=356 \mathbf{m m}$ ) used to calculate $M_{0}=0.335$.

Flow profiles were measured at three axial locations using the 9-probe system shown in fig. 4. A contour plot of one of these profiles is provided in fig. 6. This plot shows $M(y, z)$ for the case of $M_{0}=0.335$, as measured at $x=356 \mathrm{~mm}$ (middle of the lined portion of the duct). The flow is observed to be reasonably symmetric across the duct at this location. Table 1 contains the numerical values of $M(y, z)$ for this test condition. As stated in the "Test Description" section earlier, an average Mach number across the duct cross-section was achieved using a numerical integration process ( 0.335 for this case). This process was repeated at each of the three axial locations (to get three average Mach numbers), and the results were averaged to get the average Mach number $\left(M_{0}\right)$ used in the impedance eduction computations.

Acoustic measurements acquired with a two-microphone rotating plug in the side-wall of the GIT were used to determine the normal incidence impedance of the termination section $\left(\zeta_{e}\right)$. This impedance was computed using the average Mach number as an input. Table 2 provides the exit impedances for each of the Mach numbers included in this investigation at frequencies of 500 to $3000 \mathrm{~Hz}$, in steps of $500 \mathrm{~Hz}$. The corresponding exit impedances for the remaining test frequencies (tests were conducted at $100 \mathrm{~Hz}$ increments from 500 to $3000 \mathrm{~Hz}$ ) can be provided upon request. These exit impedances were assumed to hold constant for each source sound pressure level tested (120, 130 and $140 \mathrm{~dB}$ ). This is believed to be a reasonable assumption, given that the GIT termination is very nearly anechoic.

Tables 3 through 8 provide the complex acoustic pressures (SPL and phase) measured with the 31 microphones located on the wall opposite the CT57 liner for each of the Mach numbers of interest. Again, to limit the paper length, only the results for a source SPL of $130 \mathrm{~dB}$, at $500 \mathrm{~Hz}$ increments, are provided herein. These measured acoustic pressure distributions were used in the two impedance eduction codes (2D-FEM and Q3D-FEM) to determine the acoustic impedance of the CT57 liner. Figure 7 provides a comparison of the impedances (all 26 frequencies) educed with the two codes from the acoustic pressure data at $M_{0}$ 's of $0.000,0.255$ and 0.400 . In this figure, Q3D-FEM results are depicted with solid lines and 2D-FEM results are depicted with dashed lines. Blue, red and green lines are used for $M_{0}$ 's of $0.000,0.255$ and 0.400 , respectively. A subset of these impedances is also provided in table 9 . Since both impedance eduction codes are based on the 2-D convected Helmholtz equation, their respective results should be identical. However, because of the use of different programming approaches (e.g., 2D-FEM uses a band solver, Q3DFEM uses a sparse solver), their results are not identical. This difference is most noticeable near the anti-resonance (ranges from approximately $2100 \mathrm{~Hz}$ at $M_{0}=0.000$ to $1900 \mathrm{~Hz}$ at $M_{0}=0.400$ ). Given the sensitivity of the data near anti-resonance, this is not unexpected. In general, the results are very nearly identical. This excellent agreement between the two methodologies demonstrates the suitability of the quasi-3D methodology for test conditions where the 2-D method is appropriate (where a plane wave source is used), and increases confidence that the quasi-3D approach will be suitable in the presence of higher-order modes.

As stated above, the two impedance eduction codes are nearly identical for almost all frequencies away from antiresonance. It should be noted, however, that there is also significant disparity (at least on a percentage basis) at $500 \mathrm{~Hz}$, even at lower Mach numbers. Although not shown in the tabulated data (table 9), much better agreement is observed 

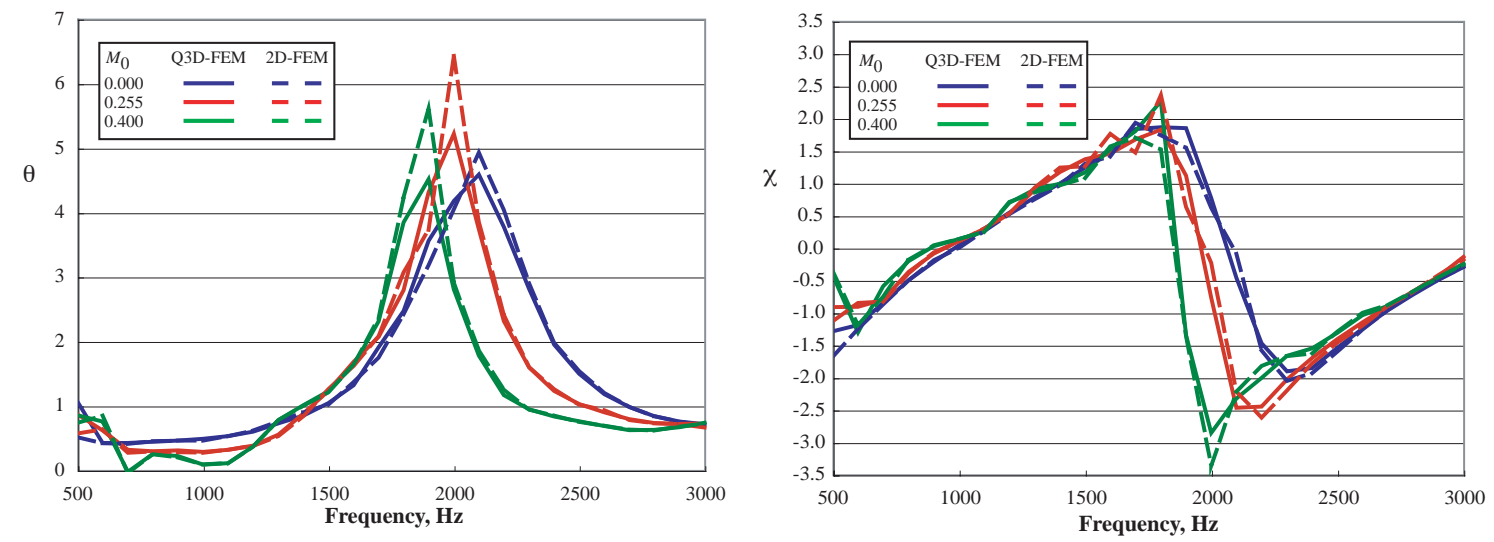

Figure 7. Normalized Impedance, $\theta+I \chi$, for CT57 Liner at $M_{0}$ of $\mathbf{0 . 0 0 0 ,} 0.255$ and 0.400 .

for frequencies at or above $600 \mathrm{~Hz}$. It should also be noted that the SPL pattern, $\operatorname{SPL}(x)$, generally contains a more linear decay for frequencies away from $500 \mathrm{~Hz}$. Together, these observations lend credence to the plausibility that the previously reported difficulties ${ }^{5}$ at $500 \mathrm{~Hz}$ are due to a longitudinal resonance.

Additional computations were performed with slight variations to the exit impedance, $\zeta_{e}$. As might be expected for a longitudinal resonance, the educed impedances were found to be extremely sensitive to the value of $\xi_{e}$ that was used as input to the impedance eduction code. Variations in the exit impedance by as little as $0.02 \rho c$ caused the educed impedance to vary by as much as $0.5 \rho c$, depending upon the Mach number tested. As a result, additional tests with a finer frequency resolution near $500 \mathrm{~Hz}$ are planned, together with more detailed exit impedance and Mach number sensitivity studies, to more fully understand the $500 \mathrm{~Hz}$ results. Tests with shorter liners will also be conducted to determine if this "resonance" condition transitions to a correspondingly higher frequency.

Finally, it should be noted that the authors are currently studying the effects of various approaches used to describe the hardwall-to-softwall interface condition. Results from this investigation will be the subject of a future paper.

\section{Concluding Remarks}

N extensive database has been acquired using the NASA Langley Research Center Grazing Incidence Tube. Mean flow profiles and complex acoustic pressure distributions were acquired for a range of parameters:

1. Source plane SPL's of 120, 130 and $140 \mathrm{~dB}$

2. Average Mach numbers of $0.000,0.079,0.172,0.255,0.335$ and 0.400

3. Test frequencies of 500 to $3000 \mathrm{~Hz}$, in steps of $100 \mathrm{~Hz}$

A portion of this data is provided in the current paper, and the remainder is available from the authors upon request.

These data were used as input to two impedance eduction codes based upon the 2-D convected Helmholtz equation. The first code (2D-FEM) is based on the 2-D assumption, whereas the second (Q3D-FEM) is a quasi-3D approach that is capable of handling higher-order modes in the vertical dimension (from hard wall to soft wall in the Grazing Incidence Tube). However, for the current investigation, only the plane wave mode was considered in the quasi-3D approach. Thus, these two methods should provide the same educed impedances. Comparisons of the results from the two methods show excellent agreement, except near the anti-resonance and at $500 \mathrm{~Hz}$. Additional analysis of the $500 \mathrm{~Hz}$ data indicates a longitudinal resonance is the cause for variability in the educed impedance results. Multiple tests, experimental and analytical, are planned to more fully understand this frequency regime.

Given the fact that the 2D-FEM has been previously demonstrated to provide quality results, the excellent comparison of the Q3D-FEM and 2D-FEM results indicates the Q3D-FEM can be used with confidence for cases where the spanwise mode order is limited to $m=0$. This enhanced code has been used with a thorough set of data measured in the GIT to provide a database that is believed to be of sufficient quality for use by others to evaluate propagation codes and impedance eduction methodologies. It is expected that these propagation codes (and correspondingly, impedance eduction methods) should increase in accuracy as the complete 3-D aeroacoustic field is included, and it is the intent of the authors to strive for this goal. 


\section{References}

${ }^{1}$ Armstrong, D.L., Beckemeyer, R.J., and Olsen, R.F., "Impedance Measurements of Acoustic Duct Liners With Grazing Flow," Paper presented at the 87th Meeting of the Acoustical Society of America, New York, NY, 1974.

${ }^{2}$ Watson, W.R., Tanner, S.E., and Parrott, T.L., "Optimization Method for Educing Variable-Impedance Liner Properties," AIAA Journal, Vol. 36, No. 1, 1998, pp. 18-23.

${ }^{3}$ Watson, W.R., Tracy, M.B., Jones, M.G. and Parrott, T.L., "Impedance Eduction in the Presence of Shear Flow," AIAA 2001-2236, "Proceedings of the 7th AIAA/CEAS Aeroacoustics Conference \& Exhibit," May, 2001.

${ }^{4}$ Jones, M.G., Parrott, T.L., and Watson, W.R., "Comparison of Acoustic Impedance Eduction Techniques for Locally-Reacting Liners," AIAA Paper 2003-3306, "Proceedings of the 9th AIAA/CEAS Aeroacoustics Conference \& Exhibit," May 12-14, 2003.

${ }^{5}$ Jones, M.G., Watson, W.R., Tracy, M.B., and Parrott, T.L., "Comparison of Two Waveguide Methods for Educing Liner Impedance in Grazing Flow," AIAA Journal, Vol. 42, No. 2, 2004, pp.232-240.

${ }^{6}$ Aurgan, Y., Leroux, M., and Pagneux, V., "Measurement of Liner Impedance with Flow by an Inverse Method," AIAA Paper 2004-2838, "Proceedings of the 10th AIAA/CEAS Aeroacoustics Conference," May 10-12, 2004.

${ }^{7} \mathrm{Li}, \mathrm{X}$., and Thiele, F., "Numerical Computation of Sound Propagation in Lined Ducts by Time-Domain Impedance Boundary Conditions," AIAA Paper 2004-2902, "Proceedings of the 10th AIAA/CEAS Aeroacoustics Conference," May 10-12, 2004.

${ }^{8}$ Elnady, T., and Boden, H., "An Inverse Analytical Method for Extracting Liner Impedance from Pressure Measurements," AIAA Paper 2004-2836, "Proceedings of the 10th AIAA/CEAS Aeroacoustics Conference," May 10-12, 2004.

${ }^{9}$ Malmary, C., Carbonne, S., Auregan, Y., and Pagneux, V., "Acoustic Impedance Measurement with Grazing Flow," AIAA Paper 2001-2193, "Proceedings of the 7th AIAA/CEAS Aeroacoustics Conference \& Exhibit," May, 2001.

${ }^{10}$ Myers, M.K., "On the Acoustic Boundary Condition in the Presence of Flow," Journal of Sound and Vibration, Vol. 71, No. 3, 1980, pp.429-434.

${ }^{11}$ Jones, M.G., and Stiede, P.E., "Comparison of Methods for Determining Specific Acoustic Impedance," Journal of Acoustical Society of America, Vol. 101, No. 5, 1997, pp.2694-2704.

${ }^{12}$ Bendat, J.S. and Piersol, A.G., "Random Data: Analysis and Measurement Procedures,” Wiley-Interscience, 1971.

${ }^{13}$ Jones, M.G., Watson, W.R., Parrott, T.L., and Smith, C.D., "Design and Evaluation of Modifications to the NASA Langley Flow Impedance Tube," AIAA Paper 2004-2837, "Proceedings of the 10th AIAA/CEAS Aeroacoustics Conference," May 10-12, 2004. 
Table 1. Measured Flow Profile, $M(y, z)$, at $x=356 \mathrm{~mm}$; Average Mach number, $M_{0}$, of 0.335

\begin{tabular}{|c|c|c|c|c|c|c|c|c|c|}
\hline & \multicolumn{10}{|c|}{$\mathbf{m m}$} \\
\hline $\mathbf{y} \mathbf{~ m m}$ & 1.3 & 6.4 & 12.8 & 19.1 & 25.4 & 31.8 & 38.1 & 44.5 & 49.5 \\
\hline 50.0 & 0.197 & 0.242 & 0.263 & 0.264 & 0.270 & 0.275 & 0.270 & 0.251 & 0.212 \\
49.0 & 0.226 & 0.264 & 0.283 & 0.294 & 0.293 & 0.295 & 0.294 & 0.276 & 0.232 \\
48.0 & 0.236 & 0.283 & 0.299 & 0.305 & 0.309 & 0.313 & 0.312 & 0.294 & 0.243 \\
46.7 & 0.243 & 0.297 & 0.314 & 0.315 & 0.323 & 0.324 & 0.326 & 0.314 & 0.257 \\
45.7 & 0.248 & 0.302 & 0.319 & 0.326 & 0.329 & 0.330 & 0.331 & 0.321 & 0.257 \\
44.5 & 0.256 & 0.307 & 0.325 & 0.335 & 0.335 & 0.338 & 0.338 & 0.323 & 0.259 \\
43.2 & 0.255 & 0.314 & 0.334 & 0.341 & 0.347 & 0.348 & 0.348 & 0.327 & 0.268 \\
40.6 & 0.251 & 0.316 & 0.342 & 0.352 & 0.361 & 0.361 & 0.357 & 0.335 & 0.269 \\
38.1 & 0.249 & 0.314 & 0.344 & 0.367 & 0.372 & 0.370 & 0.363 & 0.335 & 0.265 \\
35.6 & 0.256 & 0.318 & 0.351 & 0.374 & 0.386 & 0.384 & 0.368 & 0.333 & 0.271 \\
33.0 & 0.261 & 0.322 & 0.360 & 0.381 & 0.400 & 0.394 & 0.374 & 0.336 & 0.273 \\
30.5 & 0.265 & 0.324 & 0.359 & 0.392 & 0.405 & 0.398 & 0.372 & 0.336 & 0.269 \\
27.9 & 0.272 & 0.327 & 0.361 & 0.397 & 0.410 & 0.401 & 0.374 & 0.334 & 0.272 \\
25.4 & 0.273 & 0.335 & 0.368 & 0.397 & 0.414 & 0.405 & 0.378 & 0.339 & 0.278 \\
22.9 & 0.272 & 0.336 & 0.369 & 0.400 & 0.411 & 0.399 & 0.376 & 0.344 & 0.278 \\
20.3 & 0.275 & 0.332 & 0.366 & 0.397 & 0.402 & 0.391 & 0.371 & 0.341 & 0.275 \\
17.8 & 0.270 & 0.334 & 0.366 & 0.388 & 0.395 & 0.383 & 0.371 & 0.342 & 0.280 \\
15.2 & 0.265 & 0.334 & 0.364 & 0.381 & 0.383 & 0.374 & 0.365 & 0.343 & 0.286 \\
12.7 & 0.266 & 0.330 & 0.356 & 0.372 & 0.369 & 0.360 & 0.352 & 0.338 & 0.284 \\
10.2 & 0.272 & 0.327 & 0.345 & 0.358 & 0.357 & 0.349 & 0.345 & 0.330 & 0.280 \\
7.6 & 0.260 & 0.323 & 0.340 & 0.343 & 0.349 & 0.337 & 0.334 & 0.325 & 0.261 \\
6.4 & 0.254 & 0.316 & 0.332 & 0.339 & 0.340 & 0.328 & 0.328 & 0.321 & 0.253 \\
5.1 & 0.253 & 0.307 & 0.321 & 0.332 & 0.331 & 0.318 & 0.320 & 0.312 & 0.246 \\
4.1 & 0.249 & 0.304 & 0.312 & 0.321 & 0.328 & 0.314 & 0.315 & 0.306 & 0.247 \\
2.8 & 0.234 & 0.290 & 0.304 & 0.310 & 0.313 & 0.297 & 0.303 & 0.300 & 0.237 \\
1.8 & 0.223 & 0.272 & 0.285 & 0.298 & 0.294 & 0.276 & 0.282 & 0.282 & 0.226 \\
0.8 & 0.205 & 0.246 & 0.256 & 0.275 & 0.272 & 0.259 & 0.257 & 0.248 & 0.212 \\
\hline
\end{tabular}

Table 2. Normalized Exit Impedances, $\zeta_{e}$, for the NASA Grazing Incidence Tube

\begin{tabular}{|c|cc|cc|cc|cc|cc|cc|}
\hline & \multicolumn{2}{|c|}{$M_{0}=0.000$} & \multicolumn{2}{|c|}{$M_{0}=0.079$} & \multicolumn{2}{c|}{$M_{0}=0.172$} & \multicolumn{2}{c|}{$M_{0}=0.255$} & \multicolumn{2}{c|}{$M_{0}=0.335$} & \multicolumn{2}{c|}{$M_{0}=0.400$} \\
\hline Freq, Hz & $\theta$ & $\chi$ & $\theta$ & $\chi$ & $\theta$ & $\chi$ & $\theta$ & $\chi$ & $\theta$ & $\chi$ & $\theta$ & $\chi$ \\
\hline 500 & 0.99 & 0.06 & 1.01 & 0.05 & 0.99 & -0.10 & 0.89 & 0.21 & 0.85 & -0.13 & 0.84 & 0.21 \\
1000 & 1.02 & 0.06 & 1.03 & 0.04 & 0.94 & 0.03 & 0.93 & -0.08 & 1.09 & 0.28 & 0.95 & -0.13 \\
1500 & 1.03 & 0.08 & 1.11 & 0.03 & 1.01 & 0.05 & 1.10 & -0.09 & 0.86 & -0.17 & 1.23 & 0.03 \\
2000 & 0.98 & 0.13 & 1.11 & 0.11 & 1.05 & -0.03 & 1.04 & 0.15 & 1.04 & 0.24 & 0.89 & 0.14 \\
2500 & 1.02 & 0.11 & 1.13 & -0.01 & 1.03 & -0.13 & 0.91 & -0.09 & 1.00 & -0.20 & 0.92 & -0.14 \\
3000 & 1.00 & 0.13 & 1.16 & -0.04 & 0.89 & -0.06 & 1.04 & -0.12 & 0.95 & 0.16 & 1.29 & -0.16 \\
\hline
\end{tabular}


Table 3. Measured SPL (dB) \& Phase (deg) for CT57 liner $\left(M_{0}=0.000,130 \mathrm{~dB}\right.$ source)

\begin{tabular}{|c|c|c|c|c|c|c|c|c|c|c|c|c|}
\hline $\mathbf{x}, \mathbf{m m}$ & \multicolumn{2}{|c|}{$0.5 \mathrm{kHz}$} & \multicolumn{2}{|c|}{$1.0 \mathrm{kHz}$} & \multicolumn{2}{|c|}{$1.5 \mathrm{kHz}$} & \multicolumn{2}{|c|}{$2.0 \mathrm{kHz}$} & \multicolumn{2}{|c|}{$2.5 \mathrm{kHz}$} & \multicolumn{2}{|c|}{$3.0 \mathrm{kHz}$} \\
\hline & $\mathrm{dB}$ & deg & $\mathrm{dB}$ & deg & $\mathrm{dB}$ & deg & $\mathrm{dB}$ & deg & $\mathrm{dB}$ & deg & $\mathrm{dB}$ & deg \\
\hline 0.1 & 130.0 & -0.3 & 130.0 & -0.3 & 130.0 & 0.4 & 130.0 & 0.5 & 129.9 & 1.2 & 129.8 & 1.8 \\
\hline 25.5 & 130.5 & -10.2 & 128.2 & -16.0 & 129.5 & -46.0 & 130.0 & -49.5 & 129.7 & -70.8 & 130.1 & -76.1 \\
\hline 101.7 & 130.8 & -34.5 & 127.1 & -140.2 & 130.7 & -159.3 & 130.4 & 150.3 & 130.2 & 90.8 & 130.0 & 46.4 \\
\hline 203.3 & 127.1 & -84.8 & 128.3 & 163.3 & 131.5 & 39.5 & 130.3 & -59.4 & 130.9 & 195.0 & 132.3 & 85.3 \\
\hline 228.7 & 125.7 & -105.9 & 124.9 & 145.2 & 130.8 & 7.0 & 129.3 & -111.4 & 128.8 & 135.0 & 131.8 & 22.1 \\
\hline 254.1 & 125.3 & -128.6 & 121.7 & 125.3 & 130.6 & -24.8 & 129.4 & -163.0 & 126.9 & 66.7 & 131.0 & -41.9 \\
\hline 279.4 & 125.0 & -152.1 & 118.0 & 104.9 & 129.9 & -56.6 & 128.9 & 148.0 & 125.5 & -5.7 & 129.6 & -106.7 \\
\hline 304.8 & 125.1 & -174.9 & 114.0 & 84.1 & 129.0 & -89.5 & 128.0 & 95.6 & 124.4 & -77.6 & 127.9 & -171.5 \\
\hline 317.6 & 125.1 & 174.6 & 112.0 & 73.8 & 128.6 & -106.7 & 127.8 & 8 & 123.8 & -114.1 & 127.1 & 154.6 \\
\hline 330.3 & 125.2 & 164.4 & 109.9 & 63.0 & 128.3 & -124.0 & 127.7 & 40.9 & 123.3 & -149.9 & 126.2 & 121.1 \\
\hline 343.0 & 125.2 & 154.8 & 107.8 & 52.1 & 128.0 & -1 & 127.7 & 15.0 & 122.8 & 174.0 & 125.5 & 86.8 \\
\hline 355.7 & 125.3 & 145.3 & 105.7 & 40.7 & 127.7 & -158.1 & 127.4 & -9.4 & 122.2 & 138.6 & 124.6 & 54.6 \\
\hline 368.4 & 125.2 & 136.3 & 103.8 & 30.4 & 127.6 & -17 & 127.1 & -34.2 & 121.7 & 102.7 & 123.9 & 1.7 \\
\hline 381.1 & 125.1 & 127.6 & 101.7 & 17.6 & 127.3 & 169.3 & 126.6 & -60.5 & 121.2 & 66.5 & 122.8 & -12.0 \\
\hline 393.8 & 125.0 & 119.0 & 99.7 & 6.6 & 127.0 & 153.6 & 126.2 & -88.4 & 120.8 & 1.3 & 122.2 & -46.5 \\
\hline 406.5 & 124.7 & 110.5 & 97.9 & -4.6 & 126.6 & 13 & 126.0 & -117.2 & 120.4 & -3.9 & 121.5 & -81.6 \\
\hline 419.1 & 124.4 & 101.9 & 95.9 & -16.3 & 126.1 & 12 & 126.2 & -144.4 & 119.7 & -39.3 & 121.1 & -113.6 \\
\hline 431.9 & 124.0 & 92. & 94.1 & -27 & 125.6 & 10 & 126.4 & -169.4 & 119.1 & -75.4 & 120.4 & -144.9 \\
\hline 444.6 & 123.4 & 83.0 & 92.1 & -38.1 & 125.1 & & 126.3 & 167.0 & 118.7 & -112.6 & 119.3 & -176.8 \\
\hline 457.3 & 122.8 & 72.2 & 90.1 & -48 & 124.8 & & 125.9 & 143.7 & 118.5 & -147.7 & 118.3 & 148.8 \\
\hline 470.0 & 122.3 & 60.0 & 88.5 & -59.7 & 124.6 & 49.6 & 125.2 & 118.2 & 118.0 & 178.7 & 117.6 & 112.5 \\
\hline 482.7 & 121.8 & 47.1 & 86.5 & -70.2 & 124.6 & 32 & 124.7 & 90.3 & 117.4 & 144.6 & 117.4 & 79.9 \\
\hline 495.4 & 121.6 & 33.3 & 84.2 & -81.6 & 124.6 & 15.7 & 124.5 & 60.7 & 116.6 & 108.3 & 116.9 & 49.8 \\
\hline 508.1 & 121.6 & 19.1 & 82.4 & -89.3 & 124.5 & 07 & 124.7 & 33.7 & 116.0 & 71.9 & 115.6 & 21.9 \\
\hline 533.5 & 122.1 & -6.3 & 77.6 & -111.6 & 124.0 & -28.1 & 124.8 & -14.4 & 115.2 & 1.6 & 113.0 & -55.5 \\
\hline 558.9 & 123.0 & -27.1 & 70.3 & -127.2 & 122.6 & -57.9 & 123.4 & -63.8 & 113.0 & -70.0 & 114.1 & -119.5 \\
\hline 584.3 & 123.7 & -43.8 & 68.4 & -173.0 & 120.9 & -94.5 & 122.6 & -124.8 & 112.3 & -153.5 & 111.2 & -162.5 \\
\hline 609.7 & 124.0 & -57.7 & 69.8 & 134.8 & 119.9 & -138.8 & 123.5 & -178.8 & 113.1 & 130.1 & 103.2 & 120.1 \\
\hline 711.4 & 124.2 & -109.1 & 67.8 & 32.0 & 119.7 & 62.9 & 123.3 & -27.1 & 114.4 & -132.0 & 105.0 & 126.3 \\
\hline 787.5 & 124.3 & -147.8 & 67.6 & -41.7 & 120.6 & -58.3 & 123.6 & 171.1 & 114.6 & 31.2 & 106.2 & -112.9 \\
\hline 812.9 & 124.2 & -160.1 & 67.1 & -68.9 & 120.2 & -95.2 & 122.9 & 122.3 & 113.8 & -33.1 & 105.3 & 172.6 \\
\hline
\end{tabular}


Table 4. Measured SPL (dB) \& Phase (deg) for CT57 liner $\left(M_{0}=0.079,130 \mathrm{~dB}\right.$ source)

\begin{tabular}{|c|c|c|c|c|c|c|c|c|c|c|c|c|}
\hline \multirow[t]{2}{*}{$\mathbf{x}, \mathbf{m m}$} & \multicolumn{2}{|c|}{$0.5 \mathrm{kHz}$} & \multicolumn{2}{|c|}{$1.0 \mathrm{kHz}$} & \multicolumn{2}{|c|}{$1.5 \mathrm{kHz}$} & \multicolumn{2}{|c|}{$2.0 \mathrm{kHz}$} & \multicolumn{2}{|c|}{$2.5 \mathrm{kHz}$} & \multicolumn{2}{|c|}{$3.0 \mathrm{kHz}$} \\
\hline & $\mathrm{dB}$ & deg & $\mathrm{dB}$ & deg & $\mathrm{dB}$ & deg & $\mathrm{dB}$ & deg & $\mathrm{dB}$ & deg & $\mathrm{dB}$ & deg \\
\hline 0.1 & 130.1 & -0.1 & 130.1 & -0.1 & 130.4 & 0.5 & 30.4 & 0.6 & 130.2 & 1.4 & 130.3 & 4.6 \\
\hline 25.5 & 130.7 & -8.7 & 128.2 & -11.3 & 129.7 & -43.6 & 30.6 & -45.2 & 129.9 & -65.9 & 130.6 & -68.8 \\
\hline 101.7 & 130.9 & -28.9 & 126.5 & -135.8 & 131.1 & -147.8 & 130.9 & 166.6 & 130.3 & 109.9 & 130.5 & 71.6 \\
\hline 203.3 & 127.2 & -74.0 & 128.3 & 181.1 & 132.1 & 62.0 & 131.1 & -25.9 & 131.2 & -126.1 & 132.9 & 133.3 \\
\hline 228.7 & 125.7 & -92.9 & 124.9 & -194.3 & 131.4 & 33.0 & 129.9 & -72.5 & 129.2 & -180.0 & 132.4 & 77.4 \\
\hline 254.1 & 125.2 & -113.9 & 121.7 & 146.0 & 31.1 & 3.2 & 129.7 & -122.1 & 127.2 & 115.7 & 131.5 & 18.8 \\
\hline 279.4 & 124.8 & -135.4 & 118.0 & 124.2 & 130.4 & -26.7 & 129.6 & -167.5 & 126.1 & 46.6 & 129.9 & -41.9 \\
\hline 304.8 & 124.9 & -156.8 & 114.3 & 102.4 & 129.6 & -57.6 & 128.8 & & 125.2 & -19.0 & 128.1 & -100.6 \\
\hline 317.6 & 124.9 & -166.5 & 112.3 & 91.3 & 129.3 & -73.2 & 128.4 & 121.0 & 124.7 & -53.4 & 127.2 & -132.7 \\
\hline 330.3 & 125.1 & -176.0 & 110.3 & 79 & 129.0 & & 128.1 & & 124.3 & & 126.2 & -162.4 \\
\hline 343.0 & 125.2 & 175.5 & 108.3 & 68.0 & 128.8 & -105.1 & 128.2 & 70.1 & 123.9 & -120.3 & 125.4 & 164.2 \\
\hline 355.7 & 125.6 & 166.9 & 106.5 & & 28.6 & & 28.1 & & 123.6 & & 124.6 & 135.3 \\
\hline 368.4 & 125.6 & 159.4 & 104.6 & 43.2 & 128.4 & -136.2 & 128.1 & 23.8 & 123.1 & 175.0 & 123.9 & 104.0 \\
\hline 381.1 & 125.5 & 151.5 & 102.7 & 30. & 128.1 & -15 & 127.7 & -0 & 122.6 & 142.1 & 122.7 & 74.6 \\
\hline 393.8 & 125.5 & 145.1 & 101.1 & 17.6 & 128.0 & -166.7 & 127.4 & -24.6 & 122.3 & 106.7 & 121.8 & 41.2 \\
\hline 406.5 & 125.2 & 136.9 & 99.0 & & 127.4 & 178 & 126.9 & -50 & 122.0 & 75.4 & 120.9 & 10.1 \\
\hline 419.1 & 124.9 & 130.1 & 97.0 & -5.8 & 127.0 & 163.1 & 126.9 & -76.6 & 121.5 & 42.2 & 120.5 & -22.4 \\
\hline 431.9 & 124.5 & 122.1 & 95.3 & -17 & 126.5 & 147 & 126.9 & -102.4 & 121.0 & 11.0 & 119.8 & -50.0 \\
\hline 444.6 & 123.9 & 113.9 & 93.5 & -30.6 & 126.1 & 130.6 & 127.2 & -124.3 & 120.4 & -24.7 & 118.8 & -79.9 \\
\hline 457.3 & 123.3 & 103.9 & 91.4 & -40 & 125.8 & 113 & 127.0 & -147.3 & 120.2 & -57.9 & 117.5 & -109.2 \\
\hline 470.0 & 122.7 & 93.1 & 90.2 & -52.8 & 125.6 & 96.9 & 126.6 & -166.9 & 120.0 & -90.8 & 116.4 & -144.4 \\
\hline 482.7 & 122.2 & 80.6 & 87.8 & -61 & 125.5 & 80. & 125.9 & 168.0 & 119.7 & -119.6 & 116.0 & -174.5 \\
\hline 495.4 & 121.9 & 67.6 & 86.4 & -69.7 & 125.5 & 65.6 & 125.3 & 142.1 & 118.8 & -152.5 & 115.7 & 154.4 \\
\hline 508.1 & 122.0 & 53.6 & 84.0 & -84.7 & 125.3 & & 125.1 & 114.3 & 117.9 & 174.5 & 114.5 & 131.2 \\
\hline 533.5 & 122.8 & 30.4 & 80.9 & -111.5 & 124.7 & 23.3 & 125.8 & 66.8 & 117.7 & 103.8 & 111.6 & 62.7 \\
\hline 558.9 & 123.8 & 11.6 & 75.2 & -147.6 & 123.6 & & 125.1 & & 115.9 & 46.8 & 111.9 & -0.9 \\
\hline 584.3 & 124.6 & -2.2 & 72.8 & 0.1 & 122.3 & -39.2 & 123.5 & -28.5 & 114.1 & -41.3 & 110.0 & -40.7 \\
\hline 609.7 & 124.8 & -14.7 & 72.7 & 144.9 & 121.2 & -77.3 & 123.9 & -86.8 & 116.9 & -107.2 & 100.6 & -91.4 \\
\hline 711.4 & 125.3 & -63.1 & 72.5 & 50.2 & 121.4 & 134.6 & 124.7 & 82.4 & 116.5 & 13.7 & 103.9 & -78.2 \\
\hline 787.5 & 125.5 & -98.5 & 70.0 & -34.9 & 121.6 & 18.6 & 124.3 & -69.1 & 116.6 & -170.4 & 103.3 & 60.2 \\
\hline 812.9 & 125.4 & -108.6 & 68.7 & -58.9 & 121.7 & -13.3 & 124.6 & -108.7 & 117.2 & 126.0 & 104.2 & -17.7 \\
\hline
\end{tabular}


Table 5. Measured SPL (dB) \& Phase (deg) for CT57 liner $\left(M_{0}=0.172,130\right.$ dB source)

\begin{tabular}{|c|c|c|c|c|c|c|c|c|c|c|c|c|}
\hline \multirow[t]{2}{*}{$\mathbf{x}, \mathbf{m m}$} & \multicolumn{2}{|c|}{$0.5 \mathrm{kHz}$} & \multicolumn{2}{|c|}{$1.0 \mathrm{kHz}$} & \multicolumn{2}{|c|}{$1.5 \mathrm{kHz}$} & \multicolumn{2}{|c|}{$2.0 \mathrm{kHz}$} & \multicolumn{2}{|c|}{$2.5 \mathrm{kHz}$} & \multicolumn{2}{|c|}{$3.0 \mathrm{kHz}$} \\
\hline & $\mathrm{dB}$ & deg & $\mathrm{dB}$ & deg & $\mathrm{dB}$ & deg & $\mathrm{dB}$ & deg & $\mathrm{dB}$ & deg & $\mathrm{dB}$ & deg \\
\hline 0.1 & 130.0 & -0.4 & 130.2 & -0.6 & 130.1 & 0.2 & 30.1 & 0.2 & 130.0 & 0.7 & 130.0 & 4.5 \\
\hline 25.5 & 130.7 & -8.6 & 129.2 & -5.3 & 128.8 & -33.6 & 30.5 & -45.2 & 128.9 & -57.6 & 130.2 & -71.8 \\
\hline 101.7 & 131.4 & -26.3 & 123.5 & -123.5 & 130.4 & -140.1 & 130.6 & 177.2 & 129.2 & 128.2 & 130.8 & 87.9 \\
\hline 203.3 & 128.1 & -63.8 & 129.0 & 201.2 & 130.9 & 78.8 & 31.1 & -3.8 & 131.1 & -95.9 & 132.3 & 161.4 \\
\hline 228.7 & 126.6 & -80.4 & 125.7 & -170.2 & 130.7 & 51.3 & 130.3 & -46.9 & 129.2 & -143.4 & 132.7 & 109.5 \\
\hline 254.1 & 126.0 & -97.3 & 122.5 & 173.3 & 130.6 & 25.4 & 30.1 & -91.1 & 127.2 & 158.3 & 31.9 & 57.7 \\
\hline 279.4 & 125.3 & -116.7 & 118.8 & 151.3 & 130.0 & -1.0 & 129.8 & -135.8 & 126.1 & 91.9 & 130.4 & 4.9 \\
\hline 304.8 & 124.9 & -137.7 & 115.3 & 126.4 & 129.2 & -29.7 & 129.5 & & 125.6 & 30.1 & 128.6 & -51.9 \\
\hline 317.6 & 124.9 & -148.8 & 113.5 & 113.6 & 128.7 & -44.9 & 129.1 & 157.8 & 125.3 & -0.7 & 127.4 & -79.6 \\
\hline 330.3 & 125.1 & -158.7 & 111.7 & 101.9 & 128.3 & -60.8 & .8 & .7 & 124.8 & -31.7 & 126.4 & -108.7 \\
\hline 343.0 & 125.4 & -167.7 & 109.8 & 88.7 & 128.1 & -76.6 & 128.6 & 111.2 & 124.5 & -62.6 & 125.2 & -138.5 \\
\hline 355.7 & 125.7 & & 108.5 & & 128.0 & & 8.5 & & 124.2 & & 124.3 & -167.9 \\
\hline 368.4 & 126.0 & 177.1 & 106.9 & 62.3 & 128.0 & -106.5 & 128.5 & 65.4 & 123.9 & -124.0 & 123.5 & 163.2 \\
\hline 381.1 & 126.2 & 170.6 & 104.9 & 49 & 127.8 & -120.8 & 128.3 & 43. & 123.5 & -154.1 & 122.5 & 135.1 \\
\hline 393.8 & 126.3 & 164.9 & 103.7 & 37.3 & 127.9 & -132.6 & 128.6 & 21.7 & 123.6 & 174.9 & 121.8 & 105.6 \\
\hline 406.5 & 126.2 & 159.1 & 101.5 & 26. & 127.3 & -14 & 127.8 & -1 & 122.7 & 143.8 & 120.3 & 76.6 \\
\hline 419.1 & 126.0 & 153.8 & 99.9 & 13.1 & 126.9 & -160.2 & 127.7 & -24.8 & 122.6 & 112.9 & 119.6 & 46.5 \\
\hline 431.9 & 125.6 & 148 & 98.8 & & 126.2 & -175.1 & 127.5 & -48 & 122.1 & 83.2 & 118.7 & 17.8 \\
\hline 444.6 & 125.1 & 142.0 & 97.1 & -12.1 & 125.7 & 169.6 & 127.5 & -70.9 & 121.6 & 52.2 & 117.9 & -8.2 \\
\hline 457.3 & 124.5 & 135 & 94.7 & -29 & 125.4 & 153 & 127.7 & -92 & 121.4 & 21. & 116.9 & -36.1 \\
\hline 470.0 & 123.7 & 126.0 & 93.0 & -38.0 & 125.0 & 136.2 & 127.3 & -113.4 & 121.0 & -10.0 & 115.0 & -64.0 \\
\hline 482.7 & 122.9 & 116.1 & 91.9 & -42 & 124.9 & 119 & 126.9 & -134.6 & 120.9 & -39.0 & 114.2 & -96.6 \\
\hline 495.4 & 122.4 & 102.9 & 88.6 & -56.9 & 125.1 & 104.5 & 126.5 & -157.9 & 120.5 & -68.1 & 113.6 & -127.5 \\
\hline 508.1 & 122.1 & 88.8 & 88.1 & -76.5 & 125.2 & 91.0 & 126.2 & 178.6 & 119.7 & -97.3 & 113.2 & -151.0 \\
\hline 533.5 & 122.9 & 62.7 & 85.1 & -107.2 & 124.9 & 67.5 & 126.2 & 131.1 & 119.2 & -165.1 & 109.7 & 155.4 \\
\hline 558.9 & 124.3 & 43.5 & 79.3 & & 123.7 & 43.6 & 126.0 & 89.5 & 118.9 & 142.4 & 108.5 & 81.7 \\
\hline 584.3 & 125.4 & 31.4 & 78.2 & -61.8 & 121.5 & 13.2 & 124.9 & 44.6 & 115.6 & 76.9 & 107.9 & 43.8 \\
\hline 609.7 & 125.9 & 22.4 & 77.8 & 115.0 & 120.3 & -27.8 & 124.5 & -6.5 & 117.5 & -2.0 & 100.5 & 6.1 \\
\hline 711.4 & 125.6 & -24.7 & 73.7 & 46.8 & 120.2 & -167.1 & 124.7 & 169.0 & 118.0 & 138.6 & 100.5 & 32.2 \\
\hline 787.5 & 126.1 & -60.6 & 76.2 & -26.5 & 121.0 & 88 & 124.8 & 31.9 & 117.9 & -34.7 & 101.0 & -175.5 \\
\hline 812.9 & 126.3 & -70.8 & 78.7 & -46.8 & 120.6 & 56.5 & 125.0 & -12.9 & 118.6 & -94.2 & 100.4 & 113.1 \\
\hline
\end{tabular}


Table 6. Measured SPL (dB) \& Phase (deg) for CT57 liner $\left(M_{0}=0.255,130\right.$ dB source)

\begin{tabular}{|c|c|c|c|c|c|c|c|c|c|c|c|c|}
\hline \multirow[t]{2}{*}{$\mathbf{x}, \mathbf{m m}$} & \multicolumn{2}{|c|}{$0.5 \mathrm{kHz}$} & \multicolumn{2}{|c|}{$1.0 \mathrm{kHz}$} & \multicolumn{2}{|c|}{$1.5 \mathrm{kHz}$} & \multicolumn{2}{|c|}{$2.0 \mathrm{kHz}$} & \multicolumn{2}{|c|}{$2.5 \mathrm{kHz}$} & \multicolumn{2}{|c|}{$3.0 \mathrm{kHz}$} \\
\hline & $\mathrm{dB}$ & deg & $\mathrm{dB}$ & deg & $\mathrm{dB}$ & deg & $\mathrm{dB}$ & deg & $\mathrm{dB}$ & deg & $\mathrm{dB}$ & deg \\
\hline 0.1 & 130.0 & -0.6 & 30.5 & -0.4 & 130.0 & 0.0 & 30.0 & 0.2 & 129.8 & 1.1 & 130.0 & 5.4 \\
\hline 25.5 & 130.6 & -7.0 & 129.1 & -2.9 & 128.7 & -35.6 & 0.6 & -43.0 & 128.8 & -54.8 & 130.2 & -65.4 \\
\hline 101.7 & 130.5 & -23.2 & 124.9 & -122.5 & 130.4 & -129.4 & 130.7 & -171.4 & 129.3 & 142.5 & 130.6 & 107.3 \\
\hline 203.3 & 126.1 & -55.0 & 125.2 & -128.4 & 130.9 & 100.4 & 130.5 & 04 & 128.9 & -81.4 & 132.5 & 182.6 \\
\hline 228.7 & 126.4 & -92.8 & 128.2 & -169.5 & 131.1 & 72.5 & 131.1 & -4.8 & 130.7 & -89.0 & 132.7 & -182.9 \\
\hline 254.1 & 125.8 & -95.0 & 121.9 & -173.0 & 130.5 & 48.2 & 29.7 & -59.8 & 126.6 & -165.0 & 131.4 & 106.5 \\
\hline 279.4 & 125.0 & -109.2 & 118.6 & 164.1 & 129.8 & 22. & 129.6 & -103.5 & 125.8 & 132.3 & 129.5 & 55.2 \\
\hline 304.8 & 124.3 & -127.0 & 115.6 & 139.1 & 129.1 & & 29.5 & & 125.7 & 74.4 & 127.7 & 0.7 \\
\hline 317.6 & 123.8 & -137.7 & 113.7 & 126.5 & 128.7 & -20.2 & 129.0 & -164.7 & 125.2 & 45.4 & 126.6 & -26.0 \\
\hline 330.3 & 123.8 & -148.4 & 112.0 & 113.0 & 128.4 & -34.8 & 128.7 & & 124.9 & 16.7 & 125.5 & -52.8 \\
\hline 343.0 & 123.8 & -159.0 & 110.4 & 100.5 & 128.2 & -49.7 & 128.4 & 150.7 & 124.6 & -12.0 & 124.2 & -81.0 \\
\hline 355.7 & 124.3 & -168.2 & 108.8 & & 128.2 & & 3.5 & & 12 & & 123.2 & -108.6 \\
\hline 368.4 & 124.6 & -176.6 & 107.7 & 71.8 & 128.2 & -76.4 & 128.6 & 107.9 & 124.2 & -67.7 & 122.4 & -136.5 \\
\hline 381.1 & 124.9 & 176.5 & 105.9 & 60.2 & 128.0 & -89.1 & 128.5 & 88.2 & 123.7 & -97.1 & 121.3 & -163.3 \\
\hline 393.8 & 125.4 & 171.2 & 104.5 & 43.2 & 128.0 & -99.8 & 128.7 & 70.0 & 124.0 & -124.0 & 120.8 & 171.6 \\
\hline 406.5 & 125.5 & 165.6 & 103.3 & 31 & 127.2 & -113.9 & 127.7 & 47.1 & 123.2 & -15 & 118.7 & 142.2 \\
\hline 419.1 & 125.6 & 161.7 & 101.2 & 18.2 & 126.8 & -127.0 & 127.5 & 24.1 & 123.0 & 177.0 & 117.7 & 113.4 \\
\hline 431.9 & 125.5 & 157.6 & 100.8 & & 126.2 & -14 & 127.5 & 1. & 122.6 & 148 & 117.1 & 83.7 \\
\hline 444.6 & 125.1 & 153.4 & 99.1 & -5.3 & 125.6 & -158.6 & 127.6 & -21.9 & 122.0 & 118.2 & 116.0 & 58.4 \\
\hline 457.3 & 124.5 & 148 & 97.0 & -26 & 125.4 & -17 & 127.8 & -40.8 & 122.0 & 88.0 & 114.9 & 34.6 \\
\hline 470.0 & 123.7 & 142.3 & 97.2 & -37.2 & 125.4 & 168.5 & 127.5 & -59.9 & 121.9 & 61.1 & 112.8 & 6.9 \\
\hline 482.7 & 122.7 & 134.0 & 93.9 & -42. & 125.7 & 154 & 127.1 & -78.9 & 121.6 & 34.8 & 111.6 & -26.2 \\
\hline 495.4 & 121.9 & 122.9 & 93.3 & -65.6 & 125.9 & 141.5 & 126.4 & -101.7 & 120.8 & 6.4 & 111.5 & -56.6 \\
\hline 508.1 & 121.4 & 108.4 & 92.5 & -63.3 & 126.0 & 130.8 & 126.2 & -125.4 & 120.4 & -24.8 & 111.1 & -78.2 \\
\hline 533.5 & 121.8 & 78.7 & 85.1 & -83.1 & 125.1 & 109.5 & 126.9 & -171.0 & 120.8 & -81.3 & 106.8 & -129.9 \\
\hline 558.9 & 123.6 & 58.8 & 81.1 & -69.9 & 123.5 & & 126.6 & 154.5 & 118.6 & -132.5 & 107.1 & 162.5 \\
\hline 584.3 & 125.1 & 47.6 & 79.4 & 72.1 & 122.4 & 48.9 & 125.1 & 110.5 & 118.5 & 153.3 & 105.6 & 141.2 \\
\hline 609.7 & 125.8 & 37.9 & 82.3 & 130.5 & 122.1 & 13.9 & 125.3 & 57.0 & 119.2 & 95.4 & 91.6 & 125.4 \\
\hline 711.4 & 127.0 & 4.0 & 85.3 & 38.8 & 122.2 & -116.8 & 126.1 & -110.5 & 120.5 & -117.0 & 98.3 & 131.4 \\
\hline 787.5 & 126.2 & -23 & 82.2 & -16 & 121.6 & & 126.1 & 120.6 & 120.6 & 87.6 & 98.5 & -62.2 \\
\hline 812.9 & 125.6 & -33.2 & 83.6 & -43.0 & 122.3 & 114.0 & 125.6 & 83.1 & 119.2 & 32.3 & 99.0 & -129.2 \\
\hline
\end{tabular}


Table 7. Measured SPL (dB) \& Phase (deg) for CT57 liner $\left(M_{0}=0.335,130\right.$ dB source)

\begin{tabular}{|c|c|c|c|c|c|c|c|c|c|c|c|c|}
\hline \multirow[t]{2}{*}{$\mathbf{x}, \mathbf{m m}$} & \multicolumn{2}{|c|}{$0.5 \mathrm{kHz}$} & \multicolumn{2}{|c|}{$1.0 \mathrm{kHz}$} & \multicolumn{2}{|c|}{$1.5 \mathrm{kHz}$} & \multicolumn{2}{|c|}{$2.0 \mathrm{kHz}$} & \multicolumn{2}{|c|}{$2.5 \mathrm{kHz}$} & \multicolumn{2}{|c|}{$3.0 \mathrm{kHz}$} \\
\hline & $\mathrm{dB}$ & deg & $\mathrm{dB}$ & deg & $\mathrm{dB}$ & deg & $\mathrm{dB}$ & deg & $\mathrm{dB}$ & deg & $\mathrm{dB}$ & deg \\
\hline 0.1 & 130.1 & -0.6 & 30.0 & -1.1 & 130.3 & 0.1 & 30.2 & 0.5 & 129.7 & 0.5 & 130.1 & 5.3 \\
\hline 25.5 & 131.0 & -4.5 & 129.6 & -0.2 & 128.7 & -29.6 & 0.8 & -42.9 & 129.0 & -47.0 & 129.5 & -63.9 \\
\hline 101.7 & 130.9 & -12.7 & 122.8 & -110.5 & 130.7 & -121.1 & 131.1 & -162.9 & 129.3 & 158.4 & 130.8 & 119.2 \\
\hline 203.3 & 125.8 & -59.6 & 128.5 & -124.6 & 131.3 & 115.0 & 131.7 & 38.7 & 131.3 & -36.6 & 133.0 & -131.1 \\
\hline 228.7 & 125.5 & -76.1 & 125.0 & -133.3 & 130.6 & 91.4 & 130.9 & 1.7 & 129.1 & -77.5 & 132.3 & -173.3 \\
\hline 254.1 & 126.3 & -87.4 & 122.0 & -150.8 & 130.7 & 66.2 & 30.6 & -37.1 & 126.7 & -131.8 & 131.4 & 139.8 \\
\hline 279.4 & 125.6 & -95.3 & 118.5 & -171.8 & 130.2 & 43.1 & 129.9 & -77.9 & 126.0 & 167.9 & 129.6 & 92.4 \\
\hline 304.8 & 124.6 & -107.6 & 115.3 & & 129.4 & 19.2 & 30.1 & & 126.1 & 112.7 & 127.5 & 42.2 \\
\hline 317.6 & 123.7 & -115.9 & 114.3 & 148.8 & 128.7 & 6.0 & 129.8 & -136.8 & 125.7 & 85.3 & 126.2 & 15.6 \\
\hline 330.3 & 123.1 & -127.6 & 112.3 & 135.6 & 128.2 & & 9.4 & & 125.3 & 59.2 & 125.1 & -10.4 \\
\hline 343.0 & 122.7 & -139.8 & 111.1 & 117.2 & 127.9 & -23.9 & 128.8 & -176.9 & 125.0 & 31.9 & 123.9 & -37.4 \\
\hline 355.7 & 123.2 & -152.4 & 109.9 & & 127.9 & & 8.7 & & 125.0 & & 122.8 & -62.0 \\
\hline 368.4 & 123.9 & -163.0 & 108.5 & 92.5 & 128.2 & -52.5 & 128.8 & 139.6 & 124.7 & -20.8 & 121.7 & -89.8 \\
\hline 381.1 & 124.4 & -171.4 & 107.6 & 84 & 128.1 & -6 & 128.9 & 119.8 & 124.3 & -48.6 & 120.1 & -118.3 \\
\hline 393.8 & 125.5 & -176.6 & 106.6 & 65.5 & 128.3 & -74.9 & 129.4 & 103.2 & 124.7 & -73.2 & 120.1 & -142.4 \\
\hline 406.5 & 125.8 & 179.6 & 106.6 & 52 & 127.9 & -86.8 & 128.7 & 83. & 123.8 & -10 & 118.2 & -169.9 \\
\hline 419.1 & 126.2 & 87.3 & 105.0 & 34.1 & 127.5 & -96.1 & 128.4 & 63.8 & 123.8 & -128.9 & 117.0 & 164.9 \\
\hline 431.9 & 126.2 & 176.4 & 104.1 & & 126.6 & -10 & 127.8 & 40.0 & 123.3 & -156.7 & 115.3 & 133.8 \\
\hline 444.6 & 125.8 & 175.0 & 101.8 & -3.9 & 125.9 & -123.8 & 128.0 & 17.0 & 123.1 & 176.1 & 114.6 & 105.6 \\
\hline 457.3 & 125.2 & 173.6 & 99.4 & -13 & 125.4 & -14 & 128.6 & -0. & 123.1 & 149.9 & 114.3 & 84.8 \\
\hline 470.0 & 123.9 & 170.7 & 99.0 & -6.6 & 125.3 & -158.6 & 128.5 & -18.2 & 122.6 & 122.2 & 112.1 & 62.1 \\
\hline 482.7 & 122.4 & 164.5 & 98.5 & -1. & 125.6 & -174.4 & 128.2 & -34.1 & 122.5 & 96.3 & 109.4 & 32.6 \\
\hline 495.4 & 120.6 & 152.8 & 100.2 & -14.3 & 126.2 & 173.1 & 127.3 & -53.7 & 122.1 & 71.8 & 108.8 & -7.2 \\
\hline 508.1 & 119.6 & 131.9 & 100.1 & -16.5 & 126.5 & 163.5 & 126.6 & -77.9 & 121.4 & 44.8 & 109.0 & -31.0 \\
\hline 533.5 & 121.5 & 92.1 & 95.3 & -2.4 & 126.0 & 148.0 & 127.5 & -124.3 & 121.4 & -14.8 & 105.2 & -66.8 \\
\hline 558.9 & 125.0 & 77.8 & 99.1 & 57.6 & 123.8 & 126.3 & 127.7 & & 121.0 & -58.2 & 104.0 & -151.3 \\
\hline 584.3 & 126.7 & 74.9 & 101.6 & 93.9 & 121.6 & 87.9 & 125.3 & 164.1 & 117.4 & -124.6 & 104.3 & -159.3 \\
\hline 609.7 & 127.2 & 72.1 & 104.7 & 110.0 & 122.4 & 50.7 & 126.3 & 110.3 & 121.3 & 164.5 & 93.1 & -114.0 \\
\hline 711.4 & 125.3 & 38.7 & 101.3 & 49.5 & 122.5 & -71.9 & 127.2 & -42.9 & 120.5 & -17.9 & 94.0 & -81.3 \\
\hline 787.5 & 125.5 & -1.6 & 102.6 & -18 & 121.4 & -162.0 & 127.3 & -163.1 & 120.6 & -178.0 & 97.2 & 21.0 \\
\hline 812.9 & 125.9 & -12.8 & 102.1 & -28.9 & 122.6 & 167.2 & 125.6 & 158.7 & 121.9 & 137.8 & 96.1 & -51.2 \\
\hline
\end{tabular}


Table 8. Measured SPL (dB) \& Phase (deg) for CT57 liner $\left(M_{0}=0.400,130\right.$ dB source)

\begin{tabular}{|c|c|c|c|c|c|c|c|c|c|c|c|c|}
\hline \multirow[t]{2}{*}{$\mathbf{x}, \mathbf{m m}$} & \multicolumn{2}{|c|}{$0.5 \mathrm{kHz}$} & \multicolumn{2}{|c|}{$1.0 \mathrm{kHz}$} & \multicolumn{2}{|c|}{$1.5 \mathrm{kHz}$} & \multicolumn{2}{|c|}{$2.0 \mathrm{kHz}$} & \multicolumn{2}{|c|}{$2.5 \mathrm{kHz}$} & \multicolumn{2}{|c|}{$3.0 \mathrm{kHz}$} \\
\hline & $\mathrm{dB}$ & deg & $\mathrm{dB}$ & deg & $\mathrm{dB}$ & deg & $\mathrm{dB}$ & deg & $\mathrm{dB}$ & deg & $\mathrm{dB}$ & deg \\
\hline 0.1 & 130.0 & -0.3 & 29.9 & -1.7 & 130.3 & 0.2 & 29.9 & 0.2 & 130.3 & 1.1 & 130.3 & 4.8 \\
\hline 25.5 & 131.3 & -2.7 & 130.2 & 1.7 & 128.8 & -21.5 & 0.1 & -45.7 & 130.3 & -43.0 & 129.1 & -57.4 \\
\hline 101.7 & 131.8 & -2.9 & 120.7 & -98.1 & 130.4 & -114.5 & 130.5 & -161.5 & 130.1 & 169.6 & 130.9 & 126.3 \\
\hline 203.3 & 125.2 & -38.5 & 128.8 & -108.1 & 131.0 & 129.4 & 131.5 & 50.5 & 132.3 & -18.1 & 132.9 & -111.8 \\
\hline 228.7 & 124.5 & -63.9 & 125.4 & -117.5 & 130.2 & 106.2 & 130.3 & 13.0 & 130.2 & -58.2 & 132.3 & -153.5 \\
\hline 254.1 & 125.6 & -78.3 & 122.2 & -134.1 & 129.9 & 81.8 & 130.2 & -26.6 & 127.6 & -108.5 & 131.6 & 162.6 \\
\hline 279.4 & 125.9 & -86.9 & 118.0 & -157.8 & 129.4 & 56.7 & 129.8 & -62.6 & 126.7 & -168.9 & 129.5 & 117.1 \\
\hline 304.8 & 125.8 & -95.3 & 114.8 & 800 & 129.3 & & 29.3 & & 127.3 & & 127.8 & 68.4 \\
\hline 317.6 & 125.0 & -99.9 & 114.0 & 172.0 & 128.7 & 22 & 129.0 & -122.3 & 126.9 & 111.7 & 126.4 & 43.9 \\
\hline 330.3 & 124.3 & -106.9 & 112.8 & 15 & 12 & & 9.0 & & 126.6 & 86.9 & 124.9 & 19.0 \\
\hline 343.0 & 123.5 & -115.9 & 112.8 & 145.2 & 127.7 & -2.6 & 128.9 & -160.3 & 126.4 & 61.0 & 123.6 & -9.7 \\
\hline 355.7 & 123.2 & -127.0 & 111.7 & & 127.4 & & 8.9 & & 126.2 & 37.0 & 122.9 & -33.1 \\
\hline 368.4 & 123.3 & -140.6 & 111.0 & 109.5 & 127.3 & -30.6 & 128.7 & 163.1 & 126.0 & 11.4 & 121.9 & -57.8 \\
\hline 381.1 & 123.4 & -152.3 & 108.8 & 88.8 & 127.0 & -45 & 128.1 & 14 & 125.5 & -14.6 & 120.0 & -84.3 \\
\hline 393.8 & 124.7 & -160.1 & 108.3 & 64.2 & 127.5 & -56.3 & 128.6 & 122.8 & 125.8 & -38.3 & 119.8 & -110.3 \\
\hline 406.5 & 125.1 & -168.0 & 103.6 & 50 & 127. & -69 & 128.4 & 100.7 & 125.1 & -67 & 118.0 & -140.7 \\
\hline 419.1 & 125.9 & -170.5 & 101.0 & 33.8 & 127.3 & -79.4 & 128.6 & 84.3 & 125.1 & -91.8 & 117.0 & -162.5 \\
\hline 431.9 & 126.2 & -172.4 & 101.1 & 51 & 126.8 & -90 & 128.1 & 66. & 124.5 & $-11\}$ & 115.0 & 172.1 \\
\hline 444.6 & 126.1 & -172.1 & 103.3 & 38. & 126.4 & -101.6 & 127.8 & 47.4 & 124.4 & -145.6 & 113.5 & 140.0 \\
\hline 457.3 & 125.9 & -172.1 & 105.9 & 24. & 125.8 & -113.2 & 127.6 & 28.1 & 124.5 & -169.8 & 113.5 & 112.2 \\
\hline 470.0 & 124.8 & -172.0 & 107.8 & 2.1 & 124.9 & -128.3 & 127.5 & 5. & 124.0 & 163.5 & 112.2 & 89.5 \\
\hline 482.7 & 123.5 & -174.4 & 109.0 & -14.2 & 124.6 & -144.8 & 127.8 & -13 & 124.0 & 138.9 & 109.7 & 71.3 \\
\hline 495.4 & 121.4 & 88.9 & 106.8 & -26.9 & 124.6 & -160.3 & 127.7 & -30.2 & 123.5 & 115.2 & 107.1 & 36.0 \\
\hline 508.1 & 119.4 & 162.5 & 102.6 & -35.0 & 125.1 & & 127.4 & -46.9 & 122.9 & 90.0 & 107.7 & -3.9 \\
\hline 533.5 & 120.5 & 112.2 & 107.0 & 55.8 & 125.6 & 165.4 & 126.3 & -89.6 & 122.8 & 32.3 & 106.5 & -35.1 \\
\hline 558.9 & 125.1 & 96.5 & 112.2 & 58.5 & 124.9 & & 127.3 & & 122.8 & -10.7 & 102.2 & -122.7 \\
\hline 584.3 & 127.1 & 97.3 & 111.6 & 91.8 & 122.3 & 126.3 & 126.4 & -159.7 & 119.0 & -66.5 & 104.9 & -135.0 \\
\hline 609.7 & 127.7 & 96.5 & 114.8 & 118.3 & 120.3 & 86.5 & 124.6 & 150.9 & 122.1 & -143.5 & 97.4 & -77.5 \\
\hline 711.4 & 124.7 & 60.8 & 111.9 & 49.9 & 120.5 & -34.1 & 126.9 & -7.9 & 121.8 & 44.0 & 94.1 & -160.5 \\
\hline 787.5 & 126.1 & 18.9 & 113.1 & -10.0 & 121.7 & -115.8 & 127.1 & -122.7 & 122.3 & -111.1 & 96.8 & 75.2 \\
\hline 812.9 & 126.9 & 11.3 & 112.6 & -38.4 & 120.5 & -151.3 & 125.7 & -151.0 & 123.4 & -149.0 & 95.2 & -19.3 \\
\hline
\end{tabular}


Table 9. Normalized Impedances Educed using 2D-FEM and Q3D-FEM for CT57 Liner (130 dB source)

\begin{tabular}{|c|c|c|c|c|c|c|c|c|c|c|c|c|}
\hline 2D-FEM & \multicolumn{2}{|c|}{$M_{0}=0.000$} & \multicolumn{2}{|c|}{$M_{0}=0.079$} & \multicolumn{2}{|c|}{$M_{0}=0.172$} & \multicolumn{2}{|c|}{$M_{0}=0.255$} & \multicolumn{2}{|c|}{$M_{0}=0.335$} & \multicolumn{2}{|c|}{$M_{0}=0.400$} \\
\hline Freq, $\mathrm{Hz}$ & $\theta$ & $\chi$ & $\theta$ & $\chi$ & $\theta$ & $\chi$ & $\theta$ & $\chi$ & $\theta$ & $\chi$ & $\theta$ & $\chi$ \\
\hline 500 & 0.51 & -1.68 & 0.76 & -1.47 & 0.75 & -1.61 & 0.57 & -1.13 & 0.61 & -0.59 & 0.74 & -0.36 \\
\hline 1000 & 0.46 & 0.00 & 0.38 & 0.06 & 0.31 & 0.15 & 0.27 & 0.10 & 0.17 & 0.14 & 0.09 & 0.14 \\
\hline 1500 & 1.02 & 1.30 & 1.01 & 1.25 & 1.25 & 1.18 & 1.26 & 1.26 & 1.18 & 1.27 & 1.21 & 1.08 \\
\hline 2000 & 4.05 & 0.62 & 3.66 & 1.22 & 4.81 & -0.41 & 6.43 & -0.23 & 4.40 & -1.64 & 2.91 & -3.36 \\
\hline 2500 & 1.54 & -1.60 & 1.42 & -1.39 & 1.19 & -1.55 & 1.02 & -1.46 & 0.93 & -1.43 & 0.75 & -1.29 \\
\hline 3000 & 0.70 & -0.29 & 0.68 & -0.29 & 0.77 & -0.11 & 0.73 & -0.18 & 0.73 & -0.24 & 0.73 & -0.26 \\
\hline Q3D-FEM & \multicolumn{2}{|c|}{$M_{0}=0.000$} & \multicolumn{2}{|c|}{$M_{0}=0.079$} & \multicolumn{2}{|c|}{$M_{0}=0.172$} & \multicolumn{2}{|c|}{$M_{0}=0.255$} & \multicolumn{2}{|c|}{$M_{0}=0.335$} & \multicolumn{2}{|c|}{$M_{0}=0.400$} \\
\hline Freq, $\mathrm{Hz}$ & $\theta$ & $\chi$ & $\theta$ & $\chi$ & $\theta$ & $\chi$ & $\theta$ & $\chi$ & $\theta$ & $\chi$ & $\theta$ & $\chi$ \\
\hline 500 & 1.06 & -1.29 & 1.06 & -1.18 & 1.00 & -1.12 & 0.87 & -0.91 & 0.75 & -0.57 & 0.85 & -0.42 \\
\hline 1000 & 0.49 & 0.04 & 0.40 & 0.08 & 0.33 & 0.17 & 0.29 & 0.10 & 0.19 & 0.15 & 0.09 & 0.14 \\
\hline 1500 & 1.04 & 1.24 & 0.99 & 1.27 & 1.22 & 1.32 & 1.23 & 1.37 & 1.18 & 1.31 & 1.23 & 1.17 \\
\hline 2000 & 4.18 & 0.77 & 3.65 & 1.19 & 5.45 & -0.07 & 5.22 & -0.77 & 4.93 & -1.95 & 2.80 & -2.86 \\
\hline 2500 & 1.50 & -1.54 & 1.45 & -1.45 & 1.15 & -1.48 & 1.03 & -1.41 & 0.92 & -1.39 & 0.76 & -1.31 \\
\hline 3000 & 0.71 & -0.29 & 0.67 & -0.27 & 0.79 & -0.14 & 0.66 & -0.14 & 0.73 & -0.22 & 0.74 & -0.24 \\
\hline
\end{tabular}

